\title{
Meningitis tuberculosa: claves para su diagnóstico y propuestas terapéuticas
}

\author{
Martín Lasso B.
}

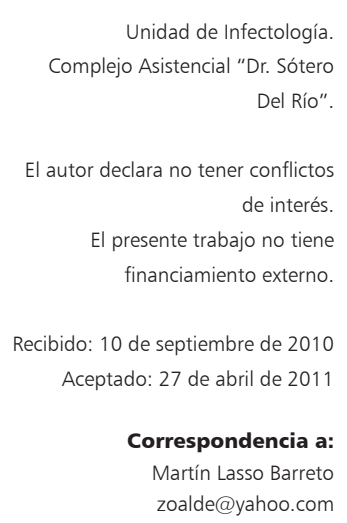

Martín Lasso Barreto zoalde@yahoo.com

\section{Tuberculous meningitis: tips for diagnosis and proposals for treatment}

Diagnosis of tuberculous meningitis (TBM) is always a challenge. We must give importance for duration of clinical manifestations. Cerebrospinal fluid (CSF) has own characteristic and it have to be control several times during the treatment. Adenosin deaminase with cut off more than $15 \mathrm{UI} / \mathrm{mL}$ and $M$. tuberculosis polymerase chain reaction in CSF are the most relevant diagnostic tests. Radiologic test gives diagnostic clues but do not confirm the diagnosis. In the future we can structure a score with all these elements to support the clinician in the diagnostic process. The treatment of TBM because of its high morbidity and high mortality has to be necessarily more intensive and prolonged and we must select drugs with a good penetration into the central nervous system (SNC). A therapeutic scheme with duration of 12 months with two phases is proposed, the diary phase during the first three months of treatment includes isoniacid, rifampicin, pirazinamid and ethambutol or moxifloxacin. Streptomycin must not be included due to own erratic SNC penetration and its known toxicity. The second twice a week phase has to be changed by a three times per week phase during 9 months and it must include isoniacid, rifampicin and pirazinamide. Dexamethasone is added during the first 6 weeks of treatment. Patients with HIV infection than required treatment with antiretroviral drugs have to start ART treatment when diary phase has finished and must not include protease or integrase inhibitors.

Key words: Tuberculous meningitis, diagnosis, adenosin deaminase, treatment.

Palabras clave: Meningitis tuberculosa, diagnóstico, adenosin deaminasa, tratamiento.

\section{Introducción}

$\mathrm{P}$ ara el año 2006 se estimaron en 9,2 millones los nuevos casos de tuberculosis (TBC) en el mundo con un incremento de su incidencia de $0,4 \%$ por año ${ }^{1}$. La meningitis tuberculosa (MTBC) representa el $1 \%$ anual de todos los casos de $\mathrm{TBC}^{2}$ y su mortalidad es elevada, llegando a ser de $30 \%$ en aquellos pacientes que reciben tratamiento óptimo ${ }^{3}$. El más importante factor que influencia en el pronóstico de la MTBC es el diagnóstico precoz y el inicio temprano de tratamiento específico ${ }^{4}$.

La mayoría de las veces en las que un médico clínico se ve enfrentado a un paciente con una probable MTBC, establecer un diagnóstico certero y buscar una terapia realmente eficiente para cada caso representa un desafío.

Considerada la forma más letal de TBC , en la práctica su diagnóstico se establece por una suma de elementos clínicos y de laboratorio que la mayoría de veces son inespecíficos, los cultivos son de crecimiento tardío y buena parte de los casos son tratadas sin un sustento microbiológico estableciéndose un "diagnóstico terapéutico" cuando existe una buena respuesta a la terapia anti TBC, lo que representa una moneda al aire para el médico tratante.

En este contexto se presenta esta revisión de las principales herramientas disponibles para el diagnóstico y las más razonables opciones de tratamiento de la MTBC; además se realizan propuestas prácticas para el manejo de estos pacientes.

\section{Diagnóstico de meningitis tuberculosa}

\section{Manifestaciones clínicas}

La presentación de la MTBC suele ser sub-aguda y habitualmente toma más de 7 días, siendo en las zonas de alta incidencia propia de individuos jóvenes ${ }^{6-8}$.

Los síntomas y signos principales, basados en las series clínicas de MTBC más grandes ${ }^{7-9}$, se señalan en la Tabla 1. Destaca la fiebre, cefalea, compromiso de conciencia, signos de irritación meníngea e hiponatremia. Se advierte lo inhabitual de las crisis convulsivas con sólo dos eventos descritos en la serie de Roca de un total de 29 casos de MTBC en un período de 15 años $^{9}$. La gravedad de la MTBC se clasifica dentro de tres estados, de acuerdo al método de Gordon y Parson ${ }^{10}$ los que se hallan relacionados con la duración de los síntomas previos, mortalidad y secuelas ${ }^{7}$ (Tabla 2).

Cerca de $75 \%$ de los pacientes con MTBC tienen concomitantemente TBC en otra localización ${ }^{11,12}$ y $60 \%$ puede llegar a tener hallazgos radiológicos consistentes con TBC pulmonar ${ }^{7}$. 


\section{Líquido cefalorraquídeo}

El estudio de LCR es la principal herramienta de laboratorio para el diagnóstico de MTBC. Las características propias del LCR se establecen en la Tabla 3 y están tomadas de dos series grandes de MTBC cuyo diagnóstico se confirmó mediante cultivo de LCR ${ }^{3,7}$.

Es necesario realizar un seguimiento de las características del LCR para establecer la adecuada respuesta a la terapia. Como se ve en la serie de Girgis y cols $^{7}$, se puede esperar una normalización del LCR a los 6 meses de seguimiento si el tratamiento es eficiente (Tabla 4).

Por otro lado en la serie sudafricana de Patel y cols ${ }^{13}$, en 99 pacientes (43\% con infección por VIH) con MTBC probable o confirmada mediante cultivo, y en los que se realizaba un seguimiento periódico del LCR durante el tratamiento, se determinó que es predecible una normalización "exponencial" de la glucosa y recuento de neutrófilos para el día 25 de tratamiento y una normalización más lenta o "lineal" de los linfocitos y proteínas, que ocurre a los 6 meses aproximadamente, estableciéndose un modelo predictor de cambio en el tiempo del LCR, en pacientes con MTBC.

Se ha intentado aplicar algoritmos para el diagnóstico de MTBC, asociando el cuadro clínico con los hallazgos del LCR; en este sentido, Török y cols ${ }^{6}$, construyen un índice en base a una serie de 205 pacientes seronegativos para VIH, con meningitis y glucorraquia baja, para diferenciar al momento de la admisión entre MTBC, meningitis bacteriana aguda (MBA) o meningitis no TBC, en áreas de alta incidencia de MTBC (Tabla 5). Este índice logra establecer, mediante regresión logística, una sensibi- lidad de 99\%, (IC95\%: 93-100\%) especificidad de 81,5\% (IC95\%: 73-88\%), un VPP de 80\% (IC95\%: 71-87\%) y un VPN de 99\% (IC95\%: 94-100\%) cuando se busca diferenciar una MTBC de una MBA, y una sensibilidad de 99\% (IC95\%:93-100\%), especificidad 68\% (IC95\%: 59-75\%), un VPP de 65,5\% (IC95\%: 56-73\%) y un VPN

Tabla 1. Características clínicas de la meningitis tuberculosa en diversas series clínicas

\begin{tabular}{|c|c|c|c|}
\hline & Girgis $\mathbf{N I}^{7}$ & Enberg $\mathbf{M}^{8}$ & Roca $B^{9}$ \\
\hline N & 857 (a) & $53(f)$ & 29 \\
\hline Origen & Egipto & Chile & España \\
\hline Años de seguimiento & 20 & 8 & 15 \\
\hline Porcentaje VIH (+) & NR & $30 \%$ & $52 \%$ \\
\hline Edad promedio & $17(b)$ & 41 & 34 \\
\hline Compromiso del estado mental (\%) & 96 (c) & $66(g)$ & 69 \\
\hline Fiebre (\%) & 90 & 76 & 93 \\
\hline Signos meníngeos (\%) & 68 (d) & 80 & 55 \\
\hline Cefalea (\%) & 63 & 68 & 69 \\
\hline Baja de peso (\%) & 54 & NR & NR \\
\hline Compromiso pares craneales (\%) & $50(e)$ & 8 & 17 \\
\hline Hiponatremia (\%) & NR & NR & 45 \\
\hline Vómitos (\%) & 49 & 53 & 38 \\
\hline
\end{tabular}

a) Todos los pacientes fueron confirmados por cultivo; b) Se incluyeron niños $(51 \%<13$ años); c) Grado II o con déficit focal $34 \%$ y grado III o estado comatosos $62 \%$; d) Rigidez de nuca $68 \%$ y signos de Kerning y Brudzinsky 65\%; e) VI:35\%, III: $10 \%$ y VII:5\%; f) $45 \%$ de pacientes confirmados por cultivo; g) Desorientado $49 \%$ y sopor $17 \%$. NR: No referido.

\begin{tabular}{|c|c|c|c|c|c|}
\hline \multirow[t]{2}{*}{ Grado } & \multirow[t]{2}{*}{ Manifestaciones clínicas } & \multicolumn{2}{|c|}{ Duración de los síntomas: } & \multirow[t]{2}{*}{ Mortalidad } & \multirow[t]{2}{*}{ Recuperación sin secuelas } \\
\hline & & 14 a 28 días & $>28$ días & & \\
\hline 1 & Vigil y orientado & $52 \%$ & $45 \%$ & $18 \%$ & $68 \%$ \\
\hline$\|$ & Somnolencia y signos neurológicos focales & $30 \%$ & $65 \%$ & $34 \%$ & $53 \%$ \\
\hline III & Coma o respuesta discreta a estímulos intensos & $21 \%$ & $74 \%$ & $72 \%$ & $14 \%$ \\
\hline
\end{tabular}

Tabla 3. Características del análisis citoquímico del LCR en la meningitis tuberculosa

\begin{tabular}{|lll|}
\hline & Girgis $\mathbf{~ N}^{\mathbf{7}}$ & Thwaiters $\mathbf{G E}^{3}$ \\
$\mathrm{~N}$ & 857 & 142 \\
\hline Celularidad/mm & $437(30-900)$ & $300(70-1.090)$ \\
\hline Glucosa $\mathrm{mg} / \mathrm{dL}$ & $22(5-55)$ & --- \\
\hline Glucosa $\mathrm{LCR} / \mathrm{sangre}$ & ---- & $0,28(0,11-0,52)$ \\
\hline Proteínas $\mathrm{g} / \mathrm{L}$ & $2,2(2,14-7,8)$ & $1,9(0,8-4,9)$ \\
\hline LCR: líquido cefalorraquídeo. & \\
\hline
\end{tabular}

Tabla 4. Evolución del LCR en meningitis tuberculosa durante el tratamiento anti-TBC ${ }^{7}$

\begin{tabular}{lcccc} 
Parámetro & Admisión & a $\mathbf{1}$ mes & a 3 meses & a $\mathbf{6}$ meses \\
\hline Leucocitos/mm & $437 \pm 347$ & $324 \pm 120$ & $124 \pm 60$ & $20 \pm 15$ \\
Glucosa, $\mathrm{mg} / \mathrm{dL}$ & $22 \pm 15$ & $41 \pm 20$ & $63 \pm 10$ & $72 \pm 5$ \\
Proteínas $\mathrm{g} / \mathrm{L}$ & $2,2 \pm 1,2$ & $1,72 \pm 1,25$ & $0,88 \pm 0,20$ & $0,30 \pm 0,10$
\end{tabular}

Valor normal: leucocitos $<10 / \mathrm{mm}^{3}$, glucosa $>65 \mathrm{mg} / \mathrm{dL}$ y proteínas $<0,28 \mathrm{~g} / \mathrm{L}$. LCR: líquido cefalorraquídeo. 


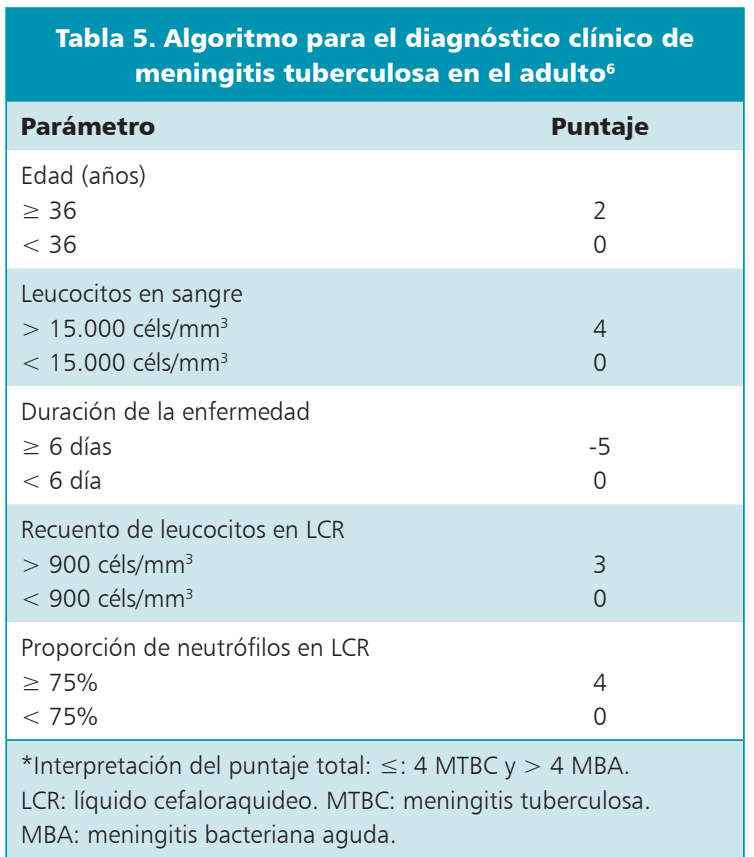

99\% (IC95\%: 94-100\%) cuando se busca diferenciar una MTBC de una meningitis no TBC. En este estudio, el estándar de oro para el diagnóstico de MTBC fue la baciloscopia o cultivo positivo para $M$. tuberculosis en el LCR. En concreto, tiene su rol para plantear que una TBC meníngea es poco probable y tiene poca utilidad para sugerirla. Sin embargo cuando se aplicó el mismo índice en zonas con alta incidencia de infección por VIH no demostró utilidad ${ }^{14}$.

\section{Estudios específicos en el LCR}

Baciloscopias. La búsqueda en visión microscópica directa de bacilos ácido alcohol resistentes en el LCR correspondientes a Mycobacterium tuberculosis, resulta muy variable; en la serie nacional de Enberg y cols ${ }^{8}$, fue negativa en todos los casos (n:0/53) y por otro lado, en un estudio reciente tuvo una sensibilidad de 53\% (IC95\%: 40,1-65,9; n: 30/57) teniendo como estándar de oro el cuadro clínico (n: 48 cultivo positivo y 7 manifestaciones clínicas, laboratorio sugerente y respuesta positiva a terapia $)^{15}$. De todas maneras se trata de una técnica sencilla de realizar y requeriría teóricamente de una carga 10.000 microorganismos por $\mathrm{mL}$ para ser positiva ${ }^{11}$. Al igual que con las muestras de expectoración, es posible incrementar su sensibilidad en LCR concentrando la muestra ${ }^{16}$.

Cultivos. El método clásico de cultivo en medio sólido de Lowestein-Jensen (LJ) o Middellbrook requiere entre tres y ocho semanas para el crecimiento de $M$. tuberculosis lo cual lo invalida como herramienta práctica para el manejo temprano de estos pacientes.

Los medios líquidos requieren un tiempo de una a tres semanas y son notoriamente más sensibles que los medios clásicos, como lo demuestra una serie india de 256 cultivos positivos de LCR de pacientes con MTBC, que en el medio líquido BACT 460TB ${ }^{\circledR}$ detectó 237 (93\%) de cultivos positivos versus 101 (39\%) para LJ, recalcando sí que es ideal poder realizar los dos cultivos ${ }^{17}$. Existen factores que se asocian a una mayor probabilidad de obtener cultivos positivos como: ser paciente con SIDA, encontrarse en fase temprana de la MTBC y tener una mayor cantidad de neutrófilos y proteínas en el LCR ${ }^{18}$.

El método de observación microscópica de susceptibilidad a drogas (MODS de sus siglas en inglés) es una técnica rápida, sensible, barata (costo por muestra 0,53 dólares) y entrega además información de susceptibilidad dentro de la primera semana del cultivo. Recientemente se ha publicado una experiencia aplicando MODS en el diagnóstico de MTBC en un centro vietnamita ${ }^{15}$. En este trabajo se analizaron 150 muestras de LCR de 137 pacientes con sospecha clínica de MTBC admitidos en un lapso de cinco meses; se realizó baciloscopias, cultivo rápido en medio líquido y cultivo de LJ. En 57 pacientes el diagnóstico final fue MTBC, en 9 de ellos el estudio microbiológico fue negativo pero no se aisló ningún otro microorganismo, las manifestaciones clínicas y el LCR fueron altamente sugerentes de MTBC, existió respuesta a la terapia antituberculosa o tenían concomitantemente TBC pulmonar. En 48 (84\%) pacientes se confirmó MTBC microbiológicamente (baciloscopias o cultivos positivos); de ellos 37, tuvieron MODS positivo. Aplicando el criterio global (clínico y microbiológico), es decir aplicando un denominador de 57, la sensibilidad de MODS fue de 64,9\% (IC95\%: 52,7-77,3; n: 37/57) y la sensibilidad, tanto del cultivo en medio líquido como del LJ, fue de 70,2\% (IC95\%: 58-82; n: 40/57), la especificidad de todos los métodos microbiológicos fue de 100\%. (LR-: 0,351 MODS, LR-:0,298 otros cultivos). Para MODS el VPP fue de $100 \%$ para diagnosticar MTBC tanto en pacientes con y sin infección por VIH y el VPN fue de $78,7 \%$ en pacientes portadores de VIH y $82,1 \%$ en no portadores de VIH.

El tiempo promedio para obtener el cultivo positivo con MODS fue de seis días lo que lo sitúa como una herramienta muy útil en las decisiones terapéuticas. En Chile no se dispone de MODS por lo que su implementación, que es sencilla, debería ser promovida.

Adenosin deaminasa (ADA). Es una enzima catalizadora para la deaminación de adenosina a inosina y amonio; existen dos isoenzimas ADA 1 y ADA2, codificadas por diferentes locus genéticos. La isoenzima ADA1 se localiza en células de alta actividad como linfocitos 
y monocitos y el ADA2 está presente principalmente en monocitos ${ }^{19}$. La ADA se ha utilizado ampliamente para el diagnóstico de MTBC, estableciéndose un corte $>7$ UI en LCR para sugerir la presencia de infección acti$\mathrm{va}^{20}$. Los estudios sobre ADA aplicados para la MTBC son muy heterogéneos y se ha definido en una revisión sistemática ${ }^{21}$ una razón de riesgo diagnóstica (DOR diagnostic odds ratio) de 65,63 (95\% IC: 11,39-378,22) con una sensibilidad de $86,5 \%$ y una especificidad de 90,5\% (LR+: 9,10/; LR-: 0,15). Sin embargo, además del las clásicas causas de incremento de ADA: MTBC, MBA y linfomas, algunas series han establecido elevación de ADA en diversas infecciones y patologías no infecciosas que comprometen el sistema nervioso central (SNC) como la neurosífilis donde hasta un tercio de los pacientes tienen ADA elevada en $\mathrm{LCR}^{22}$, la infección por $\mathrm{CMV}^{23}$ o meningitis por Criptococcus $\mathrm{sp}^{24}$. Una mención especial requiere el lupus eritematoso sistémico (LES) con compromiso del SNC que puede ser muy parecido a la MTBC en su presentación clínica, con compromiso del sensorio, manifestaciones neurológicas similares, con excepción de las crisis convulsivas, que son más frecuentes en LES y alteraciones de LCR parecidas. Esta enfermedad se presenta con ADA elevada en sangre, especialmente la isoenzima $2^{25}$, se caracteriza además por alteraciones de la barrera hemato-encefálica ${ }^{26}$ y por ello podrían incrementar los valores de esta enzima en el LCR, aunque este aspecto no ha sido estudiado específicamente.

Dado que la ADA en la TBC proviene principalmente de monocitos y macrófagos, se ha postulado que en la MTBC la isoenzima ADA 2 predomina respecto de la ADA 1, Schutte y cols ${ }^{19}$ demostraron, en una serie pequeña pero con diagnóstico microbiológico confirmado, que la relación ADA2/ADATotal > de 0,8 era altamente sugerente de MTBC respecto de MBA. Esta relación fue $>0,8$ en 14/15 pacientes con MTBC y < 0,8 en 10/11 pacientes con MBA (n: 15) (p: 0,001).

Por último, se ha propuesto que un incremento en el punto de corte de ADA a valores $>10$ o $>15$ UI podría ser útil cuando se busca mayor especificidad de esta prueba $^{27-29}$ con ello se eliminaría gran cantidad de falsos positivos y la ADA adquiriría un rol más confirmatorio de la MTBC.

Biología molecular en el diagnóstico de MTBC. Las técnicas de biología molecular han sido estimadas como las herramientas capaces de detectar la presencia de $M$ tuberculosis en todas las muestras que han sido positivas, usando el cultivo como el estándar de oro ${ }^{30}$. Sin embargo, esta apreciación no es exacta y la sensibilidad y especificidad de esta técnica es muy variable y depende de factores como una óptima extracción del ADN, si se realiza el procedimiento "en casa" ("in house”) o con un kit comercial y la correcta aplicación de los pasos para la reacción de polimerasa en cadena (RPC); todo ello relacionado con la experiencia del operador

Existen diversas secuencias de ADN específicas de $M$ tuberculosis que pueden ser amplificadas como la IS986, Ag85, MPB-64, TRC4, 65 kDa y 38 kDa ${ }^{31}$. Sin embargo, la IS6110 ha demostrado ser el ensayo de RPC de un solo paso de mejor sensibilidad y especificidad ${ }^{32-34}$.

En un estudio indio reciente ${ }^{34}$ se demostró que la RPC en casa IS6110 para TBC fue positiva en 91,4\% (n: 32/35) de pacientes con MTBC, confirmada por cultivo, y en 62,5\% (n: 10/16) de TBMC con cultivo negativo pero con un cuadro clínico, LCR compatible y respuesta terapéutica satisfactoria. Utilizando el cultivo positivo como estándar de oro, se demostró un sensibilidad de 82,4\%, una especificidad de 75,9\%, con un VPP de 85,7\% y un VPN de $71 \%$.

En un análisis sistemático de los tests de amplificación de ácidos nucleicos para el diagnóstico de $\mathrm{MTBC}^{21}$ se establece que son altamente específicos (96,2\%) pero poco sensibles (58,6\%) (LR+: 15,42 / LR-: 0,43). Sin embargo, existe mucha heterogeneidad entre los diversos tests de amplificación respecto de su sensibilidad, especialmente en aquellos en casa respecto de los kit comerciales, de los cuales parece ser el de mejor rendimiento el AMTD ${ }^{\circledR}$ con una sensibilidad de $74 \%$ y una especificidad de $98 \%$ (LR+: 37 / LR-: 0,265). En esta misma revisión se entrega una tabla que señala la probabilidad de diagnosticar MTBC al conocerse la probalilidad pre-test de la prueba aplicada, comparando ADA con RPC (Tabla 6); ambas pruebas tienen una probabilidad pre-test superior a 0,70. Concluye este análisis que la mejor prueba de laboratorio para confirmar MTBC es la RPC.

Las nuevas técnicas de RPC en tiempo real pueden llegar a ser la principal prueba diagnóstica para la MTBC en el futuro próximo ${ }^{35}$. En Chile se dispone de Amplicor MTBC $^{\circledR}$ que amplifica la secuencia de inserción IS6110 y ha sido evaluado especialmente en muestras respiratorias como muy eficiente; sin embargo, no existe la suficiente experiencia local con muestras extra-pulmonares ${ }^{36}$.

Otras pruebas diagnósticas en el LCR. Existen otras pruebas que detectan antígenos y anticuerpos para $M$. tuberculosis, con resultados variables. Se han aplicado para el diagnóstico rápido de MTBC la detección de $\gamma$ interferón derivado de células T, específico para antígenos de M. tuberculosis, que impresiona como una herramienta promisoria $^{37}$.

Otras pruebas como la deteccción del Ag 85 por la técnica de ELISA es altamente sensible; sin embargo, es comparable con la $\mathrm{ADA}^{38}$.

La determinación de anticuerpos es cuestionable como un elemento para diagnóstico, dado el paso de IgG para M. tuberculosis desde la sangre al SNC por la barrera hemato-encefálica ${ }^{39}$. Estas pruebas son inhabituales en 
Tabla 6. Probabilidad de diagnostico de meningitis tuberculosa en base a la probabilidad pre-test de ADA y RPC para M. tuberculosis en LCR (Adaptado de Dinnes J -Ref 21)

\begin{tabular}{|c|c|c|c|c|c|c|c|}
\hline \multirow[b]{2}{*}{$\begin{array}{c}\text { Probabilidad pre-test } \\
\text { de MTBC }\end{array}$} & \multicolumn{7}{|c|}{ Probabilidad post-test de MTB } \\
\hline & $\operatorname{RPC}(+)$ & RPC (-) & ADA (+) & $\begin{array}{l}\operatorname{RPC}(-) \\
\operatorname{ADA}(+)\end{array}$ & $\begin{array}{l}\text { RPC (-) } \\
\operatorname{ADA}(-)\end{array}$ & $\begin{array}{l}\operatorname{RPC}(+) \\
\operatorname{ADA}(+)\end{array}$ & $\begin{array}{l}\operatorname{RPC}(+) \\
\operatorname{ADA}(-)\end{array}$ \\
\hline 0,80 & 0,98 & 0,63 & 0,97 & 0,94 & 0,20 & 1,00 & 0,90 \\
\hline 0,60 & 0,96 & 0,39 & 0,93 & 0,85 & 0,09 & 1,00 & 0,78 \\
\hline 0,40 & 0,91 & 0,22 & 0,86 & 0,72 & 0,04 & 0,99 & 0,61 \\
\hline 0,30 & 0,87 & 0,16 & 0,80 & 0,63 & 0,03 & 0,98 & 0,50 \\
\hline 0,20 & 0,79 & 0,10 & 0,69 & 0,49 & 0,02 & 0,97 & 0,37 \\
\hline 0,10 & 0,63 & 0,05 & 0,50 & 0,30 & 0,01 & 0,94 & 0,20 \\
\hline
\end{tabular}
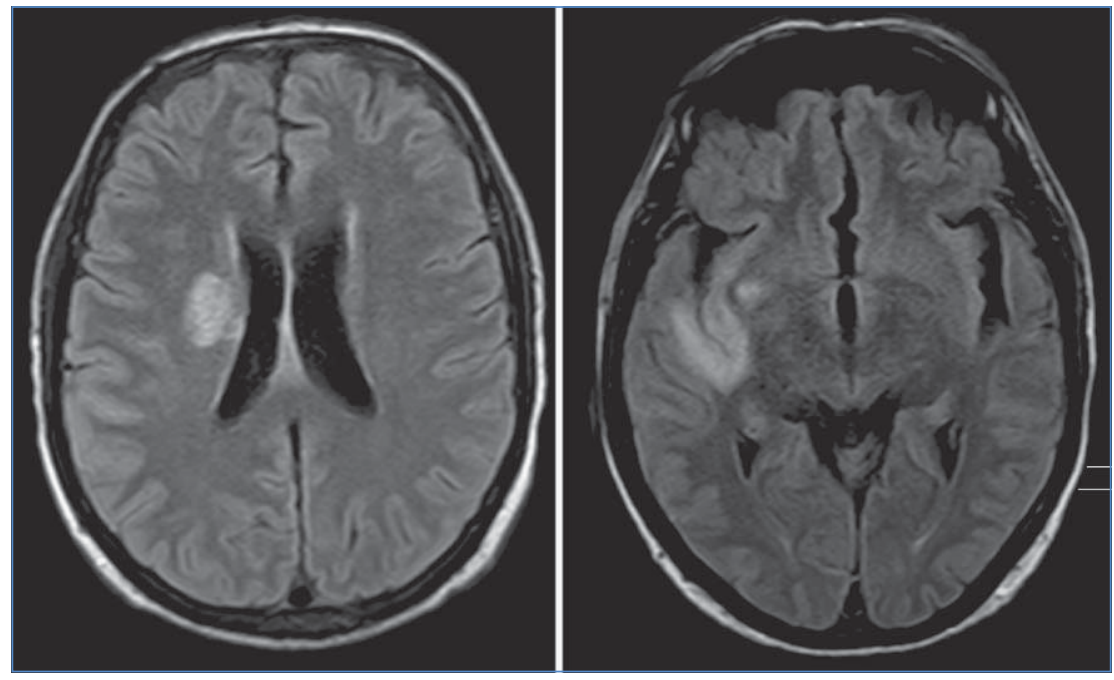

Figura 1. Imagen de resonancia magnética cerebral modo e-FLAIR de paciente con meningitis tuberculosa e infarto en la región lenticular y área insular.

nuestro medio y no son parte de los elementos usados en el diagnóstico de MTBC en Chile.

Imágenes en el diagnóstico de MTBC. Los estudios de imagen (TAC y RM cerebral) demuestran alteraciones propias de la MTBC que, en orden de frecuencia son: engrosamiento leptomeningeo o aracnoiditis, especialmente en relación al polígono de Willis y al área optoquiasmática; colecciones (abscesos o tuberculomas), infarto cerebral e hidrocefalia ${ }^{40}$. Pero este orden es variable y en una serie clínica se demostró como más habituales la hidrocefalia (51\%) y el engrosamiento meníngeo (45\%) ${ }^{41}$. Por otro lado, es importante destacar que 4 a $8 \%$ de los pacientes con MTBC seronegativos para VIH y 20\% de los seropositivos desarrollan abscesos tuberculosos los que deben ser diferenciados de los tuberculomas, los que no necesariamente se originan de una MTBC ya que pueden generarse vía hematógena de otra localización y además guardan las características de un granuloma más que de una colección propiamente tal ${ }^{41}$. La RM es el método de elección para el estudio de la MTBC; siempre debe realizarse con medio de contraste, el cual destaca los engrosamientos meníngeos, sobre todo en etapas tempranas de la infección ${ }^{42}$; por otro lado, la presencia de infartos cerebrales es más evidente en una RM y es propia de los estadios tardíos llegando a encontrarse en más de $80 \%$ de los casos en etapa III de MTBC $^{43}$. En la Figura 1 se demuestran imágenes características de la MTBC en un paciente de nuestro hospital.

Definición del diagnóstico de MTBC. Un reciente consenso $^{44}$ uniformó la definición de caso de MTBC con fines de investigación clínica estableciendo criterios clínicos de entrada y determinando tres tipos de MTBC de acuerdo al nivel de certeza: definitiva, probable y posible (Tabla 7) apoyado en un puntaje basado en la clínica, hallazgos en el LCR, imágenes cerebrales y evidencia de TBC en una localización distinta al SNC (Tabla 8). Pensando en nuestra realidad, en esta definición no se considera la ADA como un criterio, se le otorga un peso menor a los hallazgos del LCR que suelen ser muy importantes para decidir eventuales tratamientos en nuestra práctica habitual y se da un mayor peso a las imágenes, las que no siempre están disponibles.

\section{Tratamiento de la MTBC}

Terapia antituberculosa específica. La MTBC es la forma extra-pulmonar de tuberculosis de más complejo tratamiento y de más pobre respuesta. En la elección del esquema anti-TBC se debe preferir fármacos con buena penetración al SNC (Tabla 9). Isoniacida, que posee un excelente efecto bactericida, junto con pirazinamida y 


\section{Criterio clínico de entrada*}

Uno o más de los siguientes síntomas y signos: cefalea, irritabilidad, vómitos, fiebre, rigidez de nuca, convulsiones, déficit neurológico focal, alteración de conciencia y letargia

\section{Clasificación de la MTBC:}

MTBC definitiva:

Paciente debe cumplir totalmente con el criterio A o B

A) Baciloscopias positivas en LCR, cultivo o RPC comercial para M. tuberculosis positivos en LCR.

B) Baciloscopias positivas asociadas a estudio anatomo-patológico de cerebro o médula espinal compatible con MTBC y cuadro clínico y cambios en LCR sugerentes de MTBC

MTBC probable:

Puntaje diagnóstico de 10 o más (si no se dispone de imágenes cerebrales) o 12 o más (si se dispone de imágenes cerebrales) excluyendo alternativas diagnósticas

Al menos dos puntos deben provenir de hallazgos en el LCR o imágenes

MTBC posible:

Puntaje diagnóstico de 6-9 (si no se dispone de imágenes cerebrales) o de 6-11 (si se dispone de imágenes cerebrales) excluyendo alternativas diagnósticas

MTBC posible no puede ser diagnosticada ni excluida sin hacer una punción lumbar o un estudio de imagen

\section{Meningitis no tuberculosa:}

Diagnóstico alternativo establecido sin diagnóstico de MTBC definitiva y sin signos convincentes de enfermedad dual

*Debe estar presente en todas las categorías de MTBC.

\section{Tabla 8. Puntaje para sostener el diagnóstico de meningitis tuberculosa (Marais S y cols. Ref. 44)}

\section{Criterio}

Clínico

Duración de síntomas $>5$ días

Uno o más de los signos sugerentes de TBC: pérdida de peso, sudoración nocturna y tos por $>2$ semanas

Historia de contacto cercano en el último año con personas con TBC pulmonar o PPD positivo

Déficit neurológico focal

Parálisis de pares craneales

Alteración de conciencia

$L C R$

Apariencia clara

Células: 10-500/ $\mathrm{mm}^{3}$

Predominio linfocítico (> 50\%)

Proteínas mayor de $1 \mathrm{~g} / \mathrm{L}$

Relación glucosa en LCR/plasma $<50 \%$ o concentración de glucosa $<40$ mg/dL en LCR

Tuberculoma

Infarto

Hiperdensidad basal pre-contraste

\section{Puntaje}

$\mathrm{PMC}^{*}=6$

4

2

2
1

1

1

$\mathrm{PMC}^{*}=4$

Evidencia de TBC fuera del SNC

Radiografía de tórax sugerente de TBC activa: signos de $T B C=2, T B C$ miliar $=4$

TAC/RM que evidencia TBC fuera de SNC.

Baciloscopias o cultivos positivos para M. tuberculosis fuera de SNC

RPC para M. tuberculosis comercial, positiva, de muestra fuera del SNC

Exclusión de diagnósticos alternativos

Deben ser confirmados por microbiología, serología o estudio anatomo-patológico

Pensar principalmente en meningitis por bacterianas, Criptococcus spp, Treponema pallidum, virus, agentes de

la malaria o parásitos (incluyendo meningitis eosinofílica), toxoplasmosis cerebral, absceso cerebral y linfoma

*PMC: Puntaje máximo por categoría 


\begin{tabular}{|lc|}
\hline $\begin{array}{c}\text { Tabla 9. Penetración de fármacos anti } \\
\text { tuberculosos en sistema nervioso central } \\
\text { (Elaborado de acuerdo a referencias 45-49) }\end{array}$ \\
\hline Fármaco & $\begin{array}{c}\text { Paso por barrera } \\
\text { hemato-encefálica (\%) }\end{array}$ \\
\hline Isoniacida & 100 \\
\hline Pirazinamida & 100 \\
\hline Etionamida & 100 \\
\hline Rifampicina & $7-56$ \\
\hline Moxifloxacina & $50-60$ \\
\hline Levofloxacina & $30-50$ \\
\hline Etambutol & $25-50$ \\
Ciprofloxacina & $6-37(26)$ \\
\hline Amk/kan/estrp* & $0-30(20)$ \\
\hline *Amikacina, kanamicina y estreptomicina. & \\
\hline
\end{tabular}

etionamida, son fármacos de libre paso por la barrera hemato-encefálica; a consecuencia de ello, su concentración en el LCR es prácticamente similar a la sanguínea ${ }^{45-47}$. Otros fármacos tienen penetración intermedia a SNC, como moxifloxacina y levofloxacina ${ }^{48,49}$. Rifampicina, por otro lado, tiene baja penetración a SNC, el paso de este antimicrobiano ocurre en fase temprana de la MTBC y se asocia a la inflamación inicial meníngea siendo su concentración en LCR $<10 \%$ respecto de la sérica ${ }^{46}$; sin embargo, ya que la fracción libre no ligada a proteínas que corresponde al $20 \%$ de la rifampicina total ${ }^{50}$ es la que actúa sobre $M$. tuberculosis, esta condición de baja penetración es relativa desde el punto de vista terapéutico. Además, dado que más de la mitad de los casos de MTBC tienen una TBC diseminada o de otra ubicación no se puede prescindir de este fármaco en el esquema inicial $^{47}$. Etambutol y los aminoglucosidos, incluyendo estreptomicina, también penetran en fases tempranas inflamatorias del tratamiento; la concentración en el LCR de este último fármaco representa $20 \%$ de su fracción sérica ${ }^{46,47}$ de modo que su acción es marginal y posiblemente debe replantearse la indicación de estreptomicina dentro del esquema de las guías chilenas para $\mathrm{MTBC}^{51}$. Hay que recordar además que las toxicidades asociadas a aminoglucósidos son especialmente relevantes en los pacientes con MTBC y, en particular, cuando ocurre en el paciente con infección por VIH/SIDA.

El esquema que se aplica para tratamiento de MTBC en Sudáfrica, el país con mayor experiencia en el manejo de esta infección, incluye isoniacida, rifampicina, pirazinamida y etambutol, en una fase diaria de dos meses, y una terapia triple con isoniacida, rifampicina y pirazinamida tres a cinco veces a la semana en la fase de mantención, que se prolonga por seis meses ${ }^{52}$; no incluye estreptomicina en la fase diaria y se agrega pirazinamida e incrementa la frecuencia semanal en la fase de mantención. Reciente evidencia en un estudio que incluyó varios países latinoamericanos ha probado beneficio de terapias más prolongadas en la MTBC (> 6 meses) disminuyendo el riesgo de mortalidad en pacientes co-infectados por $\mathrm{VIH}^{53}$. Las guías norteamericanas de tratamiento de TBC recomiendan la prolongación del tratamiento por 9 a 12 meses en el caso de $\mathrm{MTBC}^{54}$.

El uso de corticoesteroides ha sido evaluado como útil para reducir mortalidad dentro de los primeros nueve meses ${ }^{55}$; sin embargo, recientemente se ha establecido en un estudio de seguimiento por cinco años, que su utilidad esta principalmente destinada a aquellos casos con MTBC en etapa I; no demostrando beneficio en cuanto a reducir morbi-mortalidad en la MTBC más graves ${ }^{56}$. La dosis recomendada en adultos es $12 \mathrm{mg} /$ día de dexametasona durante tres semanas y luego reducir dosis paulatinamente hasta suspender en tres semanas más ${ }^{54}$.

La respuesta a la terapia debe ser evaluada clínicamente y con sucesivas muestras de LCR para análisis citoquímico y estudio microbiológico. La toma de LCR se debe realizar luego de una semana de tratamiento, al mes y eventualmente a los 3 meses y 6 meses. Recordemos que, como ya se mencionó, la glucosa y los leucocitos deben estar normales al finalizar el primer mes de tratamiento y que a los seis meses el análisis citoquímico en su conjunto debe estar normal, siempre que exista buena respuesta a la terapia ${ }^{7,13}$.

Con respecto a los pacientes con infección por VIH/ SIDA y MTBC que deben iniciar terapia anti-retroviral (TARV), ésta debe ser aplazada para después de concluida la fase diaria del tratamiento, dado que una TARV precoz no ha demostrado beneficio en sobrevida y existe evidencia de mayor toxicidad farmacológica y fenómenos de reconstitución inmune graves ${ }^{57}$. Dicha terapia no debería incluir anti-retrovirales de la familia de los inhibidores de proteasa ni de inhibidores de integrasa debido a sus conocidas interacciones con rifampicina y que podrían llevar al fracaso de la TARV 50 .

Por último, un grave problema emergente es la presencia de $M$. tuberculosis multi-resistente y extremadamente resistentes que, si ocurren en una MTBC, incrementan significativamente la mortalidad pese al tratamiento, pudiendo alcanzar al 57 a 100\% $\%^{58-60}$. Ante esta situación se debería recurrir a fármacos de segunda línea, dando preferencia a etionamida y moxifloxacina siempre que el estudio de sensibilidad lo permita; además puede ser de utilidad el uso de terapia intratecal con amikacina y levofloxacina ${ }^{61}$.

Propuesta de tratamiento para MTBC. En la Tabla 10 se expone una propuesta inicial de tratamiento para MTBC que prolonga la fase diaria a tres meses y la fase 


\section{Infectología al Día}

de mantención a nueve meses, con un total de un año de tratamiento. Se suprime estreptomicina en la etapa diaria sustituyéndola por etambutol (eventualmente moxifloxacina), en la etapa de mantención se agrega pirazinamida al esquema con isoniacida y rifampicina y esta etapa pasa de ser bisemanal a trisemanal. Se recuerda por último, el uso de corticoides siempre en la dosificación arriba señalada, durante las primeras seis semanas.

\section{Conclusiones}

A la luz de lo revisado se concluye que el diagnostico de MTBC sigue siendo difícil y que se debe dar un peso primordial a las manifestaciones clínicas, características del LCR e, idealmente, poder contar con ADA y RPC comercial para tener una certeza diagnóstica mayor. La decisión del inicio de tratamiento debe ser consensuada pero siempre privilegiando conductas activas cuando existe una sospecha fundada de este diagnóstico, así no contemos con todos los elementos necesarios para decir que el paciente que estamos atendiendo tiene una MTBC. Al momento de tratar debemos seleccionar la mejor opción con fármacos de buena penetración al SNC, apoyándonos siempre con corticosteroides inicialmente, elaborando un esquema con cuatro fármacos al menos, intensificado y más prolongado. Si nuestro real deseo es recuperar a nuestro paciente, además lo debemos vigilar cercanamente con controles clínicos y de LCR seriados, estando atentos a la probabilidad de cepas resistentes y las toxicidades que pueden generar los fármacos antituberculosos.

\section{Resumen}

El diagnóstico de la meningitis tuberculosa (MTBC) es siempre un desafío. Debemos dar importancia a las manifestaciones clínicas y su duración. El análisis citoquímico

\begin{tabular}{|c|c|c|}
\hline Fármacos & $\begin{array}{l}\text { Fase diaria por } 3 \text { meses * } \\
\text { Dosis habitual (dosis mg/kg/día) }\end{array}$ & $\begin{array}{c}\text { Fase trisemanal por } 9 \text { meses } \\
\text { Dosis }(\mathrm{mg} / \mathbf{k g} / \mathrm{día})\end{array}$ \\
\hline Isoniacida & $300(5)^{* *}$ & $800(15)$ \\
\hline Rifampicina & $600(10)$ & $600(10)$ \\
\hline Pirazinamida & $1.500(25)$ & $1.500(50)^{* * * *}$ \\
\hline Etambutol*** & $1.200(20)$ & \\
\hline \multicolumn{3}{|c|}{$\begin{array}{l}\text { *Uso siempre de dexametasona } 4 \mathrm{mg} \text { c/8 h por } 3 \text { semanas, } \mathrm{c} / 12 \mathrm{~h} \text { en } 4^{\mathrm{a}} \text { semana, c/día en } 5^{\mathrm{a}} \text { seman } \\
\text { y día por medio en } 6{ }^{\mathrm{a}} \text { semana, luego suspender. }{ }^{* *} \text { Agregar piridoxina } 10 \mathrm{mg} / \text { día. }{ }^{* * *} \text { Moxifloxacin } \\
\text { alternativa a etambutol. }{ }^{* * * *} \text { Dosis máxima } 2.000 \mathrm{mg} .\end{array}$} \\
\hline
\end{tabular}

del LCR tiene características propias y debe ser controlado varias veces durante el tratamiento. La adenosin deaminasa con punto de corte $>15 \mathrm{UI} / \mathrm{mL}$ y la RPC para $M$. tuberculosis en LCR son las pruebas más relevantes. Las imágenes aportan elementos valiosos pero no establecen el diagnóstico por si solas. A futuro se puede estructurar un puntaje con todos estos elementos para apoyar al clínico en el proceso diagnóstico. El tratamiento de la MTBC, dada su alta morbilidad y mortalidad, necesariamente debe ser más intensivo y prolongado, y debemos seleccionar fármacos con buena penetración en el SNC. Se propone un esquema de 12 meses. La fase diaria debería durar tres meses e incluir isoniacida, rifampicina, pirazinamida y etambutol o moxifloxacina. Estreptomicina no debería ser incluida dada su mala penetración en el SNC y reconocida toxicidad. La fase de mantención debería ser trisemanal e incluir isoniacida, rifampicina y pirazinamida. Dexametasona debe administrarse durante las primeras seis semanas de tratamiento. En el caso de pacientes con infección por VIH que requieran iniciar TARV ésta debe ser aplazada para después de la fase diaria y no debería incluir inhibidores de proteasa e integrasa.

\section{Referencias}

1.- WHO Report. Global Tuberculosis Control 2008. Surveillance Planning Financing 2008 Geneva. http://www.who.int/tb/publications/ global_report/2008/pdf/fullreport.pdf (Accedido: 19 marzo 2010).

2.- Thwaites G E, Chau TT, Mai N T, Drobniewski F, Mc Adam K, Farrar J J. Tuberculosis meningitis. J Neurol Neurosurg Psychiatry 2000; 68: 289-99.

3.- $\quad$ Thwaites G E, Chau T T, Stepniewska. Diagnosis of adult TBM by use of clinical and laboratory features. Lancet 2002; 360: 1287-92.

4.- Karstaedt A S, Valtchanova S, Barriere R, Crewe-Brown H H. Tuberculosis meningitis in
South Africa urban adults. Q J Med 1998; 91: 743-7.

5.- $\quad$ Thwaiters G E, Bang N D, Dung N H, Quy HT, Oanh D T, Thoa N T. The influence of HIV infection on clinical presentation, response to treatment and outcome in adults with tuberculous meningitis. J Infect Dis 2005; 192: 2134-41.

6.- Török M E, Trung Nghia H D, Hong Chau T T, Hoang Mai N T, Thwaites G E, Stepnieska K, et al. Validation of diagnostic algorithm for adult tuberculous meningitis, Am J Trop Med Hyg 2007; 77 (3): 555-9.

7.- Girgis N I, Sultan Y, Farid Z, Mansour M M, Erian W E, Hanna LS. Tuberculous meningitis, Abbassia Fever Hospital-Naval Medical Research Unit № 3- Cairo, Egypt, from
1976-1996. Am J Trop Med Hyg 1998; 58(1): 28-34.

8.- Enberg M, Quezada M, de Toro C, Fuenzalida L. Meningitis tuberculosa en adultos: análisis de 53 casos. Rev Chil Infectol 2006; 23 (2):134-9.

9.- Roca B, Tornador N, Tornador E. Presentation and outcome of tuberculous meningitis in adults y the province of Castellon, Spain: a retrospective study. Epidemiol Infect 2008; 136: 1455-62.

10.- Gordon A, Parsons M. The place of corticosteroids in the management of tuberculous meningitis. Br J Hosp Med 1972; 7: 651-5.

11.- Fitzgerald D W, Sterling T R, Haas D W. Mycobacterium tuberculosis. En Mandell 
Douglas and Bennett's Principles and Practice of Infectious Diseases. Mandell GL, Bennett JE, Dolin R, eds. $7^{\text {th }}$ Edition 2010 Churchil Livingstone Elsevier Vol 2. 2005: 3129-63.

12.- Kennedy D H, Fallon RJ. Tuberculosis meningitis. JAMA 1974; 241: 264-8.

13.- Patel VB, Burger I, Connolly C. Temporal evolution of cerebrospinal fluid following initiation of treatment for tuberculous meningitis. S Afr Med J 2008; 98 (8): 610-3.

14.- Checkley A M, Njalale Y, Scarborough M, Zjilstra E E. Sensitivity and specificity of an index for the diagnosis of TB meningitis in patients in an urban teaching hospital in Malawi. Tropical Med Inter Health 2008; 13 (8): 1042-6.

15.- Caws M, Ha D T M, Török E, Campbell J, Thu D D A, Chau T T H, et al. Evaluation of the MODS culture technique for the diagnosis of tuberculous meningitis. PLoS ONE 2 (11): e1173. doi:10.1371/journal.pone.0001173.

16.- Callisaya J, Catacora V. Validación del método de concentración con hipoclorito de sodio para el diagnóstico de tuberculosis pulmonar. BIOFARBO 2008; 16: 54-60.

17.- Venkataswamy MM, Rafi W, Nagarathna S, Chandramuki A. Comparative evaluation of BACT 460TB system and Lowenstein-Jensen medium for the isolation of $M$ tuberculosis from cerebrospinal fluid samples of tuberculous meningitis patients. Indian J Med Microbiol 2007; 25 (3): 236-40

18.- Puccioni-Sohler M, Brandao C O. Factors associated to the positive cerebrospinal fluid culture in tuberculous meningitis. Arq Neuropsiquiatr 2007; 65 (1): 48-53

19.- Schutte C M, Ungerer J P, du Plessis H, van der Meyden. Significance of cerebrospinal fluid adenosoine deaminase isoenzymes in tuberculous (TB) meningitis. J Clin Lab Anal 2001; 15: 236-8.

20.- Baro M, Acevedo L, Lagos M E. Usefulness of adenosine deaminase determination in cerebrospinal fluid for the diagnosis of meningeal tuberculosis: 4 years experience at a public hospital. Rev Med Chil 1996; 124 : 319-26.

21.- Dinnes J, Deeks J, Kunst H, Gibson A, Cummins E, Waugh N. A systematic review of rapid diagnostic tests for detection of tuberculosis infection. Health Technology Assessment 2007; 11 (3): 83-93.

22.- Lasso M, Balcells M E, Fernández A, Gaete P, Serri M, Pérez J. Neurosífilis en pacientes portadores y no portadores de VIH. Descripción y comparación de dos cohortes históricas. Rev Chil Infectol 2009; 26 (6): 532-9.

23.- Lasso M, Pérez J, Noriega LM, Albert F, González P, Malebrán A Polirradiculopatía por citomegalovirus en 2 pacientes con SIDA: tratamiento exitoso con terapia anti retroviral de alto grado de actividad (TAAGA) Rev Med Chile 2001; 129 (9): 1061-4.

24.- Martínez E, Domingo P, Ris J, Sambeat M A, Cadafalch J. Cerebrospinal fluid adenosine deaminase levels in a patient with cryptococcal meningitis. Clin Infect Dis 1992; 15: 1061-2.

25.- Taysi S, Polat MF, Ali Sari R, Bakan E. Serum adenosine deaminase and cytidine deaminase activities in patients with systemic lupus erythematosus. Clin Chem Lab Med 2002; 40 (5): 493-5.

26.- Abbott N J, Mendonça L L F, Dolman D E M. The blood-brain barrier in systemic lupus erythematosus. Lupus 2003; 12: 908-15.

27.- Kashyap R S, Kainthla R P, Mudaliar A V, Purohit H J, Taori J M, Daginawala H F. Cerebrospinal fluid adenosine deaminase activity: A complimentary tool in the early diagnosis of tuberculous meningitis. Cerebrospinal Fluid Research 2006; 3: 5-11.

28.- Choi S H, Kim Y S, Bae I G, Chung J W, Lee M S, Kang J M. The possible role of cerebrospinal fluid adenosine deaminase activity in the diagnosis of tuberculous meningitis in adults. Clin Neurol Neurosurg 2002; 104: 10-15.

29.- Chotmongkol V, Teerajetgul Y, Yodwut C. Cerebrospinal fluid adenosine deaminase activity for the diagnosis of tuberculous meningitis in adults. Southeast Asian J Trop Med Public Health 2006; 37 (5): 948-52.

30.- Caws M, Wilson S M, Clough C, Drobniewski. Role of IS6110-target PCR, culture, biochemical, clinical and immunological criteria of diagnosis of tuberculous meningitis. J Clin Microbiol 2000; 38 (9): 3150-5.

31.- Rafi W, Vekataswamy M M, Ravi V, Akepati C. A comparative evaluation of in-house PCR assays involving three mycobacterial DNA sequences, IS6110. Rapid diagnosis of tuberculous meningitis: MPB-64 and $65 \mathrm{kDa}$ antigen. J Neurol Sci 2006; 252: 163-8

32.- Bandyopadhyay D, Gupta S, Banerjee S, Gupta S, Ray D, Bhattacharya S, et al Adenosine deaminase estimation and multiplex polymerase chain reaction in diagnosis of extrapulmonary tuberculosis. Int J Tuberc Lung Dis 2008; 12 (10): 1203-8.

33.- Rafi W, Vekataswamy M M, Nagarathna S, Satishchandra P, Akepati C. Role of an IS6110 Uniplex PCR in the diagnosis of tuberculous meningitis: Experience at a tertiary neurocentre. Int J Tub Lung Dis 2007; 11 (2): 209-14.

34.- Deshpande P S, Kashyap R S, Ramteke S S, Nagdev K J, Purohit H J, Taori G M, et al. Evaluation of the IS6110 PCR assay for the rapid diagnosis of tuberculous meningitis. Cerebrospinal Fluid Reserch 2007; 4: 10.

35.- Takahashi T, Tamura M, Asami Y, Kitamura E, Saito K, Suzuki T, et al. Novel wide-range quantitative nested real time PCR assay for Mycobacterium tuberculosis DNA: development and methodology. J Clin Microbiol 2008, 46 (5): 1708-15.

36.- Selman C, Poggi H, Román JC, García P, Lagos M. Análisis retrospectivo del rendimiento de Amplicor-PCR ${ }^{\circledR}$ para la detección de Mycobacterium tuberculosis en muestras respiratorias y no respiratorias con baciloscopia negativa. Rev Chil Infectol 2009; 26 (6): 495-8.

37.- Thomas M M, Hinks TS, Raghuraman S, Ramalingam N, Ernst M, Nau R. Rapid diagnosis of Mycobacterium tuberculosis meningitis by enumeration of cerebrospinal fluid antigen-specific T-cells. Int J Tuberc Lung Dis 2008; 12 (6): 651-7.

38.- Kashyap R, Ramteke S, Deshpande P, Purohit H, Taori G, Daginawala H. Comparasion of an adenosine deaminase assay with ELISA for the diagnosis of tuberculous meningitis infection. Med Sci Moint 2007; 13 (9): BR200-4.

39.- Restrepo B, Pino P, Volcy M, Franco A, Kanaujia G, Robledo J. Interpretation of mycobacterial antibodies in the cerebrospinal fluid of adults with tuberculous meningitis. Trop Med Int Health 2008; 13 (5): 653-8.

40.- Villoria M F, de la Torre J, Fortea F, Muñoz L, Hernández T, Alarcón J J. Intracranial tuberculosis in AIDS: CT and MRI findings. Neuroradiol 1992; 34 (1): 11-4.

41.- Whiteman M, Espinoza L, Post M J, Bell M D, Falcone S. Central nervous system tuberculosis in HIV-infected patients: clinical and radiographic findings. Am J Neuroradiol 1995; 16 (6): 1319-27

42.- Öztoprak I, Gümüs C, Öztoprak B, Engin A. Contrast medium-enhanced MRI findings and changes over time in stage I tuberculous meningitis. Clin Radiol 2007; 62: 1206-15.

43.- Shukla R, Abbas A, Kumar P, Gupta R, Jha S, Prasad KN. Evaluation of cerebral infarction in tuberculous meningitis by diffusion weighted imaging. J Infect 2008; 57: 298-306.

44.- Marais S, Thwaites G, Schoeman J, Török M, Misra U, Prasad K, et al. Tuberculous meningitis : a uniform case definition for use in clinical research. Lancet Infect Dis 2010; 10 (11): 803-12.

45.- Humphries M. The management of tuberculous meningitis (Editorial). Thorax 1992; 45: 577-81.

46.- Ellard G A, Humphries M J, Allen B W. Cerebrospinal fluid drug concentrations and the treatment of tuberculous meningitis.Am Rev Respir Dis 1993; 148: 650-5.

47.- Thwaites G E, Lan N T, Dung N H, Quy H T, Oanh D T, Thoa N T. Effect of antituberculosis drug resistance on response to treatment and outcome in adults with tuberculous meningitis. J Infect Dis 2005; 192 (1):79-88.

48.- Alffenaar J, de Vries PM, Luijckx GJ, van Soolingen D, van der Werf T S, van 
Altena R. Plasma and cerebrospinal fluid pharmacokinetics of moxifloxacin in a patient with tuberculous meningitis. Antimicrob Agents Chemother 2008; 51(6): 2293-5.

49.- Van Wart S, Bhavnani S, Chau T, Farrar J, Ambrose P,Thwaites G. Population pharmacokinetics (PPK) and cerebrospinal fluid (CSF) Penetration for fluoroquinolones (FQ) in tuberculosis meningitis (TBM) patients. $50^{\text {th }}$ ICAAC 2010 Boston September 12-15 Abstract A1-057.

50.- Sande M, Eliopoulus G, Moellering R, Gilbert D. The Sanford Guide to HIV/AIDS Therapy 2006-2007. $15^{\text {th }}$ ed. 2006: 146.

51.- Programa Nacional de Control de Tuberculosis. Manual Año 2005. Norma General Técnica № 82. Ministerio de Salud de Chile. 2005.

52.- The South African National Tuberculosis Control Programme. Practical Guidelines 2004. www.kznhealth.gov.za/.../Guidelines/ Guidelines\%20National/Tuberculosis/SA\%20 TB\%20Guidelines\%202004.pdf (Accedido 24 septiembre 2010)
53.- Cortes C, McGowan C, Wehbe F, Duda S, Shephed B, Leger P, et al. Association of TB treatment duration and intermittency with death and TB recurrence risk in HIV infected persons. Abstract 783. 17th CROI 2010. San Francisco.

54.- Centers for Disease Control and Prevention. Treatment of Tuberculosis. American Thoracic Society, CDC and Infectious Diseases Society of America. MMWR Morbid Mortal Wkly Rep 2003; 52 (No. RR-11): 57-9.

55.- Thwaites G E, Nguyen DB, Nguyen HD, Hoang T, Do Thi T, Nguyen $T$ et al. Dexamethasone for the treatment of tuberculous meningitis in adolescents and adults. N Engl J Med 2004; 351: 1741-51.

56.- Török M E, Bang N, Chau T, Yen N, Thwaites G E, Dung N et al. Dexamethasone and long-term outcome in patients with tuberculous meningitis. $49^{\text {th }}$ Interscience Conference on Antimicrobial Agents and Chemotherapy. San Francisco, CA., September 12-15, 2009. Abstract L1-436.

57.- Török M E, Yen N, Chau T, Mai N, Phu N,
Mai P. Randomised controlled trial of immediate versus deferred antiretroviral therapy in HIV associated tuberculous meningitis. $49^{\text {th }}$ Interscience Conference on Antimicrobial Agents and Chemotherapy. San Francisco, CA., September 12-15, 2009. Abstract H1224.

58.- Byrd T D. Multidrug-resistant tuberculous meningitis. Curr Neurol Neuroscience Report 2007; 7: 470-5.

59.- Patel V B, Padayatchi N, Bhigjee AI, Allen J, Bhagwan B, Moodley A, et al. Multidrugresistant tuberculous meningitis in KwaZuluNatal, South Africa. Clin Infect Dis 2004; 38: 851-6.

60.- Daikos G L, Cleary T, Rodriguez A, Fischl MA. Multidrug-resistant tuberculous meningitis in patients with AIDS. Int J Tuberc Lung Dis 2003; 7:394-8

61.- Berning S, Cherry T, Iseman. Novel treatment of meningitis caused by multidrug-resistant Mycobacterium tuberculosis with intrathecal levofloxacin and amikacin: case report. Clin Infect Dis 2001; 32: 643-6. 